\title{
Carpal tunnel syndrome: evaluation of a new method of assessing median nerve conduction at the wrist
}

\author{
C A Dunne, P W Thompson, J Cole, J Dunning, C N Martyn, D Coggon, C Cooper
}

\begin{abstract}
Objective-To compare median nerve conduction velocity measured using a new, portable electroneurometer with measurements made using conventional hospital nerve conduction apparatus.

Methods-Twenty five patients were studied who were consecutively referred to a hospital neurophysiology department with a clinical diagnosis of carpal tunnel syndrome. Sensory and motor latencies for the median nerve at the wrist were measured bilaterally using the portable electroneurometer and a Medilec MS 92 hospital apparatus operated by a trained technician.
\end{abstract}

Results-There was strong agreement between motor latency values obtained by the two techniques $(r=0.89, p<0.001$; mean difference $-0.03 \mathrm{~ms}$, limits of agreement -0.33 to $0.27 \mathrm{~ms}$ ). Sensory latencies were less easy to detect with the electroneurometer, and correlated less well with the hospital apparatus $(r=0.78, p<0.001$; mean difference $-0.16 \mathrm{~ms}$, limits of agreement -0.50 to $0.18 \mathrm{~ms}$ ).

Conclusion-The portable electroneurometer provides a convenient, rapid, and inexpensive means of assessing median nerve conduction velocity at the wrist. Measurements of motor latency obtained with this new instrument agree more strongly with those made by conventional apparatus than do measurements of sensory latency. Although the utility of the instrument in clinical practice will be limited, it provides a helpful tool in epidemiological studies of carpal tunnel syndrome.

(Ann Rheum Dis 1996; 55: 396-398)

Carpal tunnel syndrome is a clinical problem encountered commonly in neurological and rheumatological practice. During 1989/90, 18087 median nerve decompression procedures were performed in England and Wales and a large additional number of patients received non-surgical treatment in hospitals and general practice. ${ }^{1}$ In most cases the diagnosis of carpal tunnel syndrome can be made on clinical features, though the sensitivity and specificity of various clinical tests has been questioned. $^{23}$ Nerve conduction studies and electromyography help to confirm the diagnosis of carpal tunnel syndrome and provide a quantitative estimate of the severity of compression. However, current techniques for assessing median nerve conduction at the wrist are not easily applicable to epidemiological studies of carpal tunnel syndrome.

A new device, the Electroneurometer (Nerve Pace, S-200 Nerve Conduction Monitor, Neurotron Medical, Lawrenceville, New Jersey, USA), that is portable and less expensive than conventional nerve conduction instruments $(£ 3300$ compared with around $£ 15000)$ is now available. This instrument permits rapid assessment of median nerve conduction in carpal tunnel syndrome and would provide a valuable research tool in epidemiological studies of the disorder. However, its validity remains uncertain. The purpose of this study was to compare the instrument with conventional nerve conduction instruments in assessing median nerve conduction across the wrist in patients with carpal tunnel syndrome.

\section{Patients and methods}

We studied 25 patients ( 16 men, nine women) referred consecutively to a hospital neurophysiology department with a clinical diagnosis of carpal tunnel syndrome. Conventional nerve conduction studies measuring motor and sensory latencies were performed bilaterally using a Medilec MS 92 apparatus, operated by a trained technician (JD). The patients also underwent nerve conduction assessments using the electroneurometer (fig 1) (operated by CD). The electroneurometer measurement entailed a 10 minute procedure during which subjects were seated with their arm resting on a flat surface and the elbow flexed at $30^{\circ}$. The thenar eminence, wrist area, and dorsum of the hand were cleansed with alcohol and three self adhering electrodes were applied to the subject's hand. A ground electrode was placed on the dorsum of the hand and remained there throughout the entire procedure. The active electrode was placed over the belly of the abductor pollicis brevis muscle, and the reference electrode on the radial/volar aspect of the proximal phalanx of the thumb. Conductive gel was applied to the stimulating probe and the distal portion of the probe was placed over the path of the median nerve, $3 \mathrm{~cm}$ 


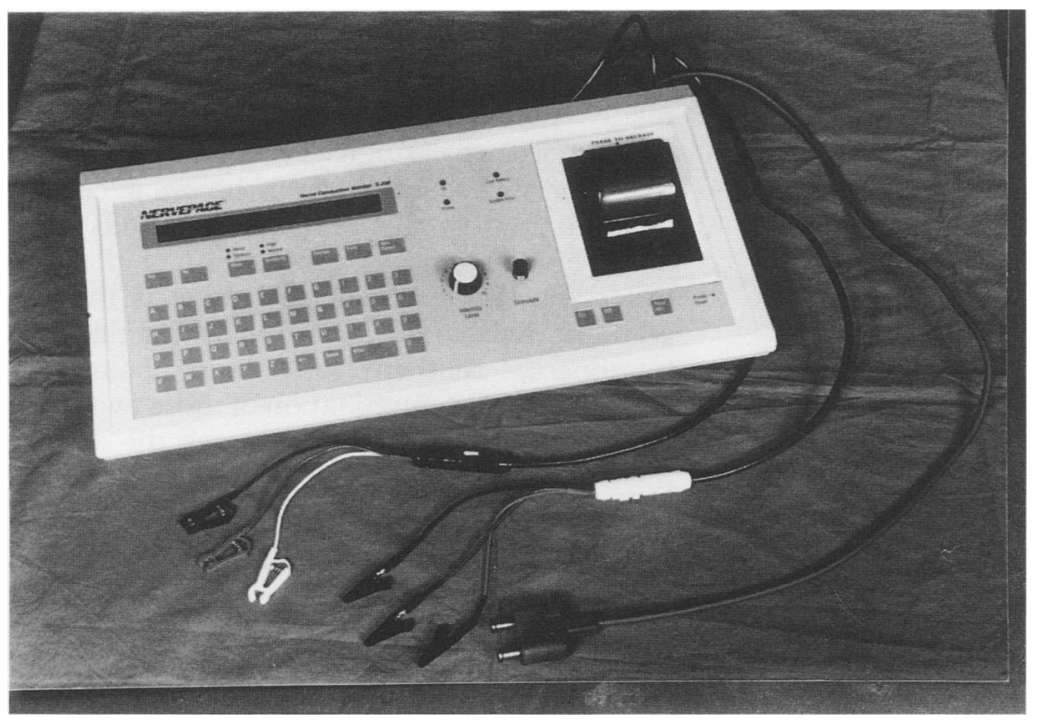

Figure 1 The portable electroneurometer.

proximal to the distal wrist crease. A low intensity stimulus was then delivered to the skin over the median nerve. Latencies were displayed digitally on the electroneurometer. Measurements were performed bilaterally, with distal motor and sensory latencies expressed in milliseconds.

Values obtained using the two techniques (electroneurometry and conventional nerve conduction measurement) were compared using Spearman rank correlation coefficients and the kappa statistic. The limits of agreement were estimated using the method of Bland and Altman. ${ }^{4}$ The coefficient of variation of the electroneurometric measurements has previously been demonstrated to be between 1 and $4 \% .^{5}$

\section{Results}

The mean age of the 25 subjects was 53.6 years (range 27-83 years). When conventional motor criteria were used to define carpal tunnel syndrome (a distal motor latency of greater

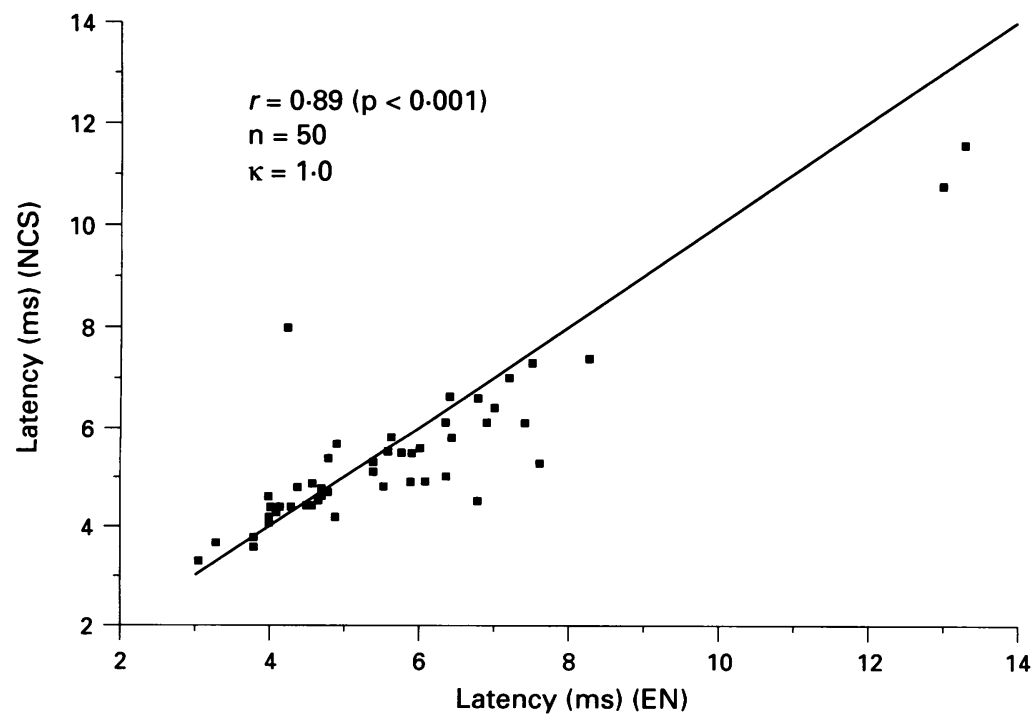

Figure 2 Comparison between median nerve motor latencies measured using the portable electroneurometer (EN), and conventional nerve conduction studies (NCS). than 4 ms using conventional neurophysiological apparatus $\left.{ }^{6}\right), 21$ of the subjects studied had conduction delay affecting one or both wrists. We found a strong positive correlation between the measurements of motor latency obtained by the two techniques $(r=0 \cdot 89$, $p<0.001$ ) (fig 2). When this threshold was used to define abnormality on the electroneurometer, there was complete diagnostic concordance $(\kappa=1)$ between the portable instrument and the conventional nerve conduction instrument. The mean difference between motor latencies on the electroneurometer and by conventional nerve conduction was $-0.03 \mathrm{~ms}$, with limits of agreement between -0.33 and $0.27 \mathrm{~ms}$.

Sensory latencies could be recorded in only 22 wrists. Agreement between the two instruments was not as good for sensory latencies $(r=0.78, \mathrm{p}<0.001)$. Figure 3 shows the greater dispersal of the readings obtained by the two methods, and there was appreciable $(7 / 22)$ misclassification between them ( $\kappa=0.32$, using a sensory threshold of $3.5 \mathrm{~ms}$ ). The mean difference between sensory latencies measured by the electroneurometer and by conventional nerve conduction was $-0.16 \mathrm{~ms}$, with limits of agreement between -0.50 and $0.18 \mathrm{~ms}$. Sensory recordings were also more difficult to obtain using the electroneurometer, and in nine subjects in whom motor latency was prolonged beyond the manufacturer's threshold, no sensory recording could be made.

\section{Discussion}

We have evaluated a new method of assessing median nerve conduction velocity at the wrist in carpal tunnel syndrome. The electroneurometer is portable, relatively inexpensive, and easy to use. The procedure is acceptable to patients and the measurements have previously been shown to be reproducible..$^{5}$ The time taken for each examination was around 10 minutes for the electroneurometer and around 15 minutes for conventional nerve conduction apparatus. Our results showed a close correlation between measurements of median nerve motor latency made using the new instrument and those made using conventional neurophysiology apparatus. The validity for measurement of sensory latency was not as great for the new instrument and, furthermore, sensory latencies were unrecordable by electroneurometer in several of our patients, usually among patients with severe carpal tunnel syndrome. However, the sensory action potential has a considerably lower amplitude than that of the motor action potential, and sensory latencies are also more difficult to elicit with conventional nerve conduction apparatus. We are aware of one previous comparison between the electroneurometer and standard nerve conduction studies. Rosecrance et $a l^{7}$ confined their study to measurement of median nerve sensory latencies in a series of subjects, using both methods. They reported a greater correlation $(r=0.92)$ than we found between the results using each method. The mean difference between matched pairs of observations, 


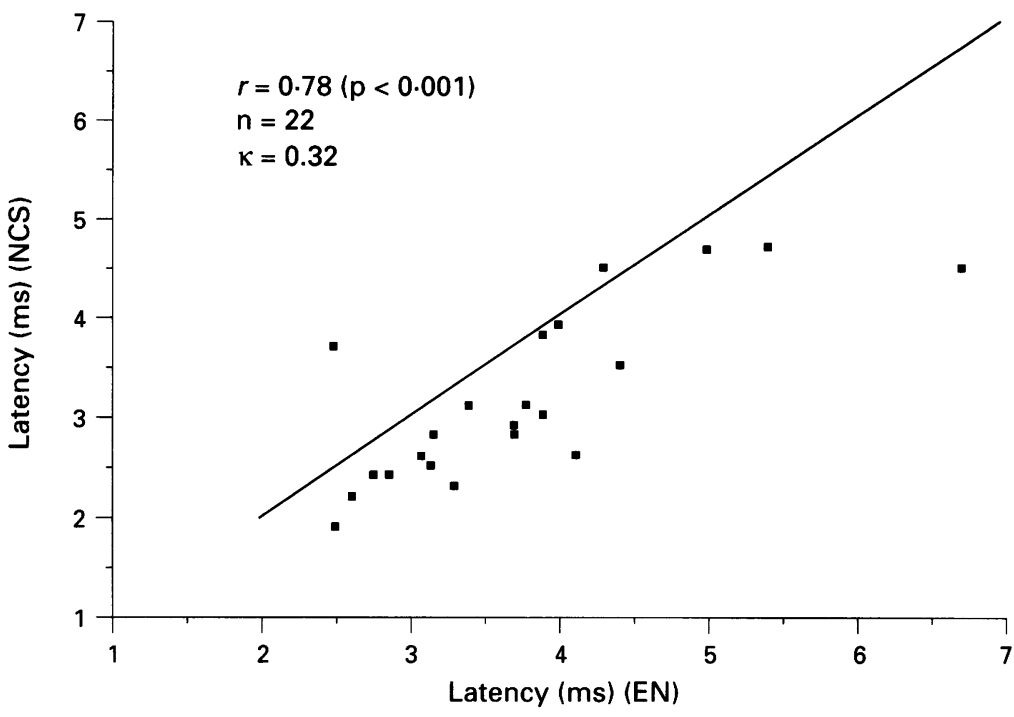

Figure 3 Comparison of median nerve sensory latencies measured using the portable electroneurometer (EN) and conventional nerve conduction studies (NCS).

however, was $0.09 \mathrm{~ms}$ - a value not significantly different from that of $0.16 \mathrm{~ms}$ in our study. The majority of their subjects were asymptomatic, and it is possible that the electroneurometer is more accurate in certain parts of the range of latency values than in others. More rigorous training for the technician might also improve the correlation we observed.

Sensory latency is considered a more useful discriminant in borderline or mild carpal tunnel syndrome; around $85 \%$ of patients with clinically significant carpal tunnel syndrome have a delayed sensory response, in contrast with $66 \%$ who have prolongation of motor latency. ${ }^{6} 8$ The poorer performance of the electroneurometer for these measurements in our hands is an important deficiency. A second potential limitation of the new technique is the absence of a visible wave form that can be examined by the operator. This is in marked contrast to conventional nerve conduction studies, in which interpretation is often enhanced by inspection of the trace obtained. Third, we measured only median digital nerve action potentials, and it has been suggested that a difference between ulnar and median values might be more accurate. ${ }^{9}$ Finally, the diagnostic utility of the electroneurometer will be limited in clinical settings if more than one nerve is affected. Thus diabetic patients with concomitant carpal tunnel syndrome and peripheral neuropathy, or those with chronic renal failure, would be better investigated by conventional nerve conduction studies.
Our study suggests that the portable electroneurometer will provide a highly specific, but relatively insensitive, tool for epidemiological studies of carpal tunnel syndrome. Its sensitivity for such studies might be enhanced by combination with alternative methods that focus on the sensory component of the disorder. The best validated of these is the self administered hand diagram in which tingling, numbness, sensory loss, and pain are recorded. ${ }^{10}$ The combination of these two techniques, and measures to improve the assessment of sensory latency using the electroneurometer, are important areas for future research.

In conclusion, our data suggest that portable electroneurometric measurement of median nerve motor latency at the wrist provides a convenient and inexpensive means of assessing the motor component of carpal tunnel syndrome, and that the measurements so obtained are closely related to those obtained by conventional hospital based neurophysiology apparatus. Carpal tunnel syndrome and other upper limb disorders are an important cause of sickness absence from work. ${ }^{11}$ Research into the relationship between various types of occupational physical activity and the risk of carpal tunnel syndrome is urgently required. Portable electroneurometry represents a means of assessing median nerve conduction in such epidemiological studies.

We are grateful to IBM Ltd for purchase of the portable electroneurometer, and to Dr P Egger for assistance with the analysis. The manuscript was prepared by Mrs Denise Gould.

1 Hospital Episode Statistics. London: Department of Health 1993.

2 Golding D N, Rose D M, Selvarajah K. Clinical tests for carpal tunnel syndrome: an evaluation. $\mathrm{Br} \mathcal{F}$ Rheumatol 1986; 25: 388-90.

3 Katz J N, Larson M G, Sabra A, et al. The carpal tunnel syndrome: Diagnostic utility of the history and physical syndrome: Diagnostic utility of the history and

4 Bland J M, Altman D G. Statistical methods for assessing agreement between two methods of clinical measurement. agreement between two

5 Cook T N, Rosecrance J C, Brokman S J, Rulon A S, Wise C A. Reliability of the digital electroneurometer fo the determination of motor latency of the median nerve. fournal of Occupational Rehabilitation 1991; 1: 105-12.

6 Binnie C D, Cooper R, Fowler C J, Maugiere F, Prior P F Osselton J W. Clinical neurophysiology: nerve conduction and evoked potentials. Oxford: Butterworth Heinemann, 1995.

7 Rosecrance J C, Cook T M, Bingham R C. Comparison of a digital electroneurometer and standard nerve conduction studies for the measurement of median nerve sensory latency. Fournal of Occupational Rehabilitation 1993; 3: 191-9.

8 Hoffman D E Carpal tunnel syndrome: Importance of sensory nerve conduction studies in diagnosis. $7 A M A$ 1975; 233: 983-4.

9 Pease W S, Cannell C D, Johnson E W. Median to radial latency difference test in mild carpal tunnel syndrome. .

$10 \mathrm{Katz}$ J N, Stirrat C R. A self administered hand diagram for the diagnosis of carpal tunnel syndrome. $\mathcal{F}$ Hand Sur 1990; 15A: 360-3.

11 Hagberg $M$, Morgenstern $H$, Kelsh $M$. Impact of occupations and job tasks on the prevalence of carpal tunnel syndrome. Scand $\mathcal{F}$ Work Environ Health 1992; 18 $337-45$. 